\title{
Determination of the light curve of the Rosetta target asteroid (2867) Steins by the OSIRIS cameras onboard Rosetta ${ }^{\star}$
}

\author{
M. Küppers ${ }^{1}$, S. Mottola ${ }^{2}$, S. C. Lowry ${ }^{3}$, M. F. A’Hearn ${ }^{4}$, C. Barbieri ${ }^{5}$, M. A. Barucci ${ }^{6}$, S. Fornasier ${ }^{5,6}$, \\ O. Groussin ${ }^{4,7}$, P. Gutiérrez ${ }^{8}$, S. F. Hviid ${ }^{1}$, H. U. Keller ${ }^{1}$, and P. Lamy ${ }^{7}$ \\ 1 Max-Planck-Institut für Sonnensystemforschung, Germany \\ e-mail: kueppers@mps.mpg.de \\ Institute of Planetary Research, DLR, Germany \\ Astrophysics Research Centre, Queen's University Belfast, UK \\ Department of Astronomy, University of Maryland, USA \\ Dipartimento di Astronomia and CISAS, Università di Padova, Italy \\ University of Paris 7, France \\ Laboratoire d'Astrophysique de Marseille, France \\ ${ }^{8}$ Instituto de Astrofísica de Andalucía - CSIC, Spain
}

Received 3 November 2006 / Accepted 30 November 2006

\section{ABSTRACT}

\begin{abstract}
Context. In 2004 asteroid (2867) Steins has been selected as a flyby target for the Rosetta mission. Determination of its spin period and the orientation of its rotation axis are essential for optimization of the flyby planning.

Aims. Measurement of the rotation period and light curve of asteroid (2867) Steins at a phase angle larger than achievable from ground based observations, providing a high quality data set to contribute to the determination of the orientation of the spin axis and of the pole direction.

Methods. On March 11, 2006, asteroid (2867) Steins was observed continuously for $24 \mathrm{~h}$ with the scientific camera system OSIRIS onboard Rosetta. The phase angle was 41.7 degrees, larger than the maximum phase angle of 30 degrees when Steins is observed from Earth. A total of 238 images, covering four rotation periods without interruption, were acquired.

Results. The light curve of (2867) Steins is double peaked with an amplitude of $\approx 0.23$ mag. The rotation period is $6.052 \pm 0.007 \mathrm{~h}$. The continuous observations over four rotation periods exclude the possibility of period ambiguities. There is no indication of deviation from a principal axis rotation state. Assuming a slope parameter of $G=0.15$, the absolute visual magnitude of Steins is $13.05 \pm 0.03$.
\end{abstract}

Key words. minor planets, asteroids - techniques: photometric

\section{Introduction}

Rosetta is a Cornerstone mission of the European Space Agency (ESA). Its goal is to monitor a comet over several months on its way to perihelion with an orbiter and to investigate its surface and interior with a lander. Additionally, flybys of 2 asteroids are part of the mission plan.

The launch of Rosetta, first planned for January 2003, had to be postponed to the first quarter of 2004 due to problems with the launcher. As a consequence, all 3 mission targets had to be changed. Comet $67 \mathrm{P} /$ Churyumov-Gerasimenko was chosen as the new target comet. After the successful launch on 2 March 2004 the asteroids (2867) Steins and (21) Lutetia were selected as flyby targets, with closest approach in Sep. 2008 and July 2010, respectively.

While the properties of the large main belt asteroid (21) Lutetia are relatively well known, (2867) Steins had not been investigated in detail before it was selected as a spacecraft target. Since knowledge of the global properties of the target is

* Table 2 is only available in electronic form at http: //www . aanda.org essential for the success of the flyby, several observing programs of Steins have been initiated starting in 2004.

Steins has been classified as an E-type asteroid based on its visual and near-infrared spectrum (Barucci et al. 2005) and on its high albedo, as determined from its polarimetric properties (Fornasier et al. 2006). The polarimetric and spectral properties of Steins imply an extensive thermal history of a differentiated body. Steins may have a composition similar to relatively rare enstatite chondrite/achondrite meteorites. With an absolute magnitude of 13.18 (Hicks et al. 2004), the polarimetric albedo of $0.45 \pm 0.10$ corresponds to a diameter of approximately $4.6 \mathrm{~km}$.

Hicks et al. (2004) reported a rotation period of $6.06 \pm 0.05 \mathrm{~h}$ for Steins, which was followed up with a full detailed analysis by Weissman et al. (2005), resulting in a more precise value of $6.048 \pm 0.007 \mathrm{~h}$ for the synodic period. The light curve amplitude on both occasions was $0.20-0.29 \mathrm{mag}$. The colours determined by Hicks et al. (2004) were $V-R=0.58 \pm 0.03$, $R-I=0.44 \pm 0.03$, and $B-V=0.80 \pm 0.03$, while Weissman et al. (2005) derived $V-R=0.51 \pm 0.03$ and $R-I=0.44 \pm 0.03$.

Here we report on observations of Steins obtained with the scientific optical camera system OSIRIS onboard Rosetta. The data set taken from a spacecraft in interplanetary space is unique 


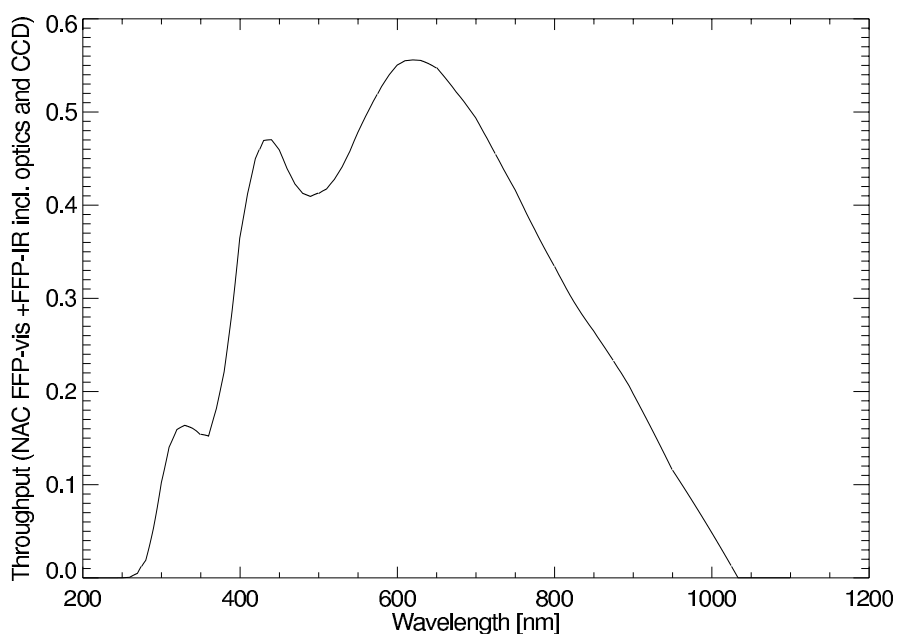

Fig. 1. System throughput of the OSIRIS NAC with clear filter, including the transmission of the mirrors and of the visual and near-infrared re-focussing plate, and the quantum efficiency of the CCD. The cut-off at near-ultraviolet wavelengths is due to the near-IR focus plate becoming opaque, the cut-off in the near-IR is caused by the decreasing quantum efficiency of the CCD.

in terms of its continuous coverage over $24 \mathrm{~h}$ and in the phase angle of 41.7 degrees, which is larger than the maximum phase angle of Steins that can be seen from Earth (30 degrees).

\section{Instrument, observations and data reduction}

OSIRIS consists of a narrow angle camera (NAC) and a wide angle camera (WAC). They are unobstructed mirror systems with focal lengths of $72 \mathrm{~cm}$ (NAC) and $14 \mathrm{~cm}$ (WAC). The focal ratio is $f / D=8$ and $f / D=5.6$ for the NAC and the WAC, respectively. Both cameras are equipped with $2048 \times 2048$ pixel CCD detectors with a pixel size of $13.5 \mu \mathrm{m}$. The image scale is $3.9 \mathrm{arcsec} / \mathrm{pixel}$ for the NAC and $20.5 \mathrm{arcsec} / \mathrm{pixel}$ for the WAC. OSIRIS is described in detail in Keller et al. (2006).

The NAC was used for the observations of (2867) Steins because of its higher point source sensitivity compared to the WAC. The NAC is equipped with two filter wheels, containing 8 positions each. This allows for numerous combinations of 11 medium bandwidth filters, 4 re-focussing plates, and a neutral density filter. For the observations of (2867) Steins the re-focussing plates for the visual and for the near-infrared were used, effectively providing a clear filter. The system throughput in this configuration is shown in Fig. 1.

The observations took place on 11 March 2006. The distance of (2867) Steins from Rosetta and from the Sun was 1.06 AU and 2.30 AU, respectively. The phase angle of Steins was 41.7 degrees. The Rosetta spacecraft tracked the expected motion of Steins. A total of 238 images with 300 s exposure time each were obtained, one image every $6 \mathrm{~min}$. A window of $512 \times 256 \mathrm{CCD}$ pixels centered on the expected position of Steins was read out.

The data were reduced with the OSIRIS standard calibration pipeline. For the Steins observations, the pipeline performed the following steps:

- The CCD is read out using a dual 14 bit Analogue to Digital Converter (ADC). DN values created by the "upper" ADC (DN values $\geq 2^{14}$ ) get an additional offset added by the read out electronics. This offset is subtracted.

- A noise filter removes coherent noise in the data produced by the power converter.
- The bias is subtracted using bias exposures taken immediately after the Steins observations.

- The images are divided by a flat field using reference flat fields from the ground calibration.

- The data are converted into units of $\mathrm{W} \mathrm{m}^{-2} \mathrm{~nm}^{-1}$ sterad $^{-1}$, using a conversion factor derived from observations of Vega, the secondary spectrophotometric standard stars $58 \mathrm{Aql}$ and $\epsilon \mathrm{Aqr}$, and of the solar analog stars $16 \mathrm{Cyg} \mathrm{A}$ and $16 \mathrm{Cyg} \mathrm{B}$. This conversion is valid for a solar source spectrum.

After the systematic processing pipeline had been applied, we carried out the photometric reduction procedure, aimed at extracting the flux information from the images. During the observation period Steins moved across the NAC field of view, and it appulsed background stars in many instances. For this reason, the first step of the photometric reduction consisted of modeling the star background and its subsequent subtraction. To this end, all of the science images were resampled, registered and co-added in five groups, to provide an accurate background representation for different segments of the asteroid's trajectory. During this process, transient spurious events ("cosmic ray hits") have been discriminated by comparison and removed. Subsequently, the corresponding background fields have been subtracted from each science frame. The subtraction in image space (as opposed to subtraction of total flux) has been made possible by the fact that the spacecraft tracking was very accurate (see Fig. 2), with an error within a single exposure smaller than the PSF of the camera. Finally, the asteroid flux was extracted from the background-subtracted images by means of a synthetic aperture procedure in which the pixel intensities are integrated over a user-defined aperture. The phase angle varied between $41.59^{\circ}$ and $41.83^{\circ}$ during the observations. Since the brightness change induced by the phase angle change is expected to be less than $0.01 \mathrm{mag}$, no phase correction has been performed. The resulting light curve is shown in Table 2 and Fig. 3.

\section{Results and discussion}

The resulting photometric time series have been converted to magnitudes and modelled as a 6th order Fourier polynomial (Harris et al. 1989a), whose best fit gave a synodic rotation period of $6.052 \pm 0.007 \mathrm{~h}$. The data folded with the best fit period are shown in Fig. 4.

The figure demonstrates that the light curve of (2867) Steins is double peaked, with an amplitude of $\approx 0.23 \mathrm{mag}$. The rotation period is $6.052 \pm 0.007 \mathrm{~h}$, which fits previous determinations well (Hicks et al. 2004; Weissman et al. 2005). The continuous observations over four rotation periods exclude the possibility of period ambiguities. The behaviour of the light curve is not symmetric and the differences between the two maxima and two minima would imply an irregular shape for the asteroid. The shoulder on the descending portion of the light curve near the second minimum is reminiscent on a similar feature in the light curve of comet 9P/Tempel 1 (A'Hearn et al. 2005). In the case of Tempel 1 the shoulder was caused by large, relatively flat surfaces abutting each other.

There is no indication of a deviation from a principal axis rotation state. Therefore, the OSIRIS data, taken together with ground based data obtained at different epochs, can be used to derive a solution for pole orientation (Lamy et al. 2006).

If the rotational brightness variation were caused by a smooth, featureless triaxial ellipsoid that scatters light geometrically, then the diameter ratio $a / b$ between the longest and the intermediate semiaxis is $>1.23$. 


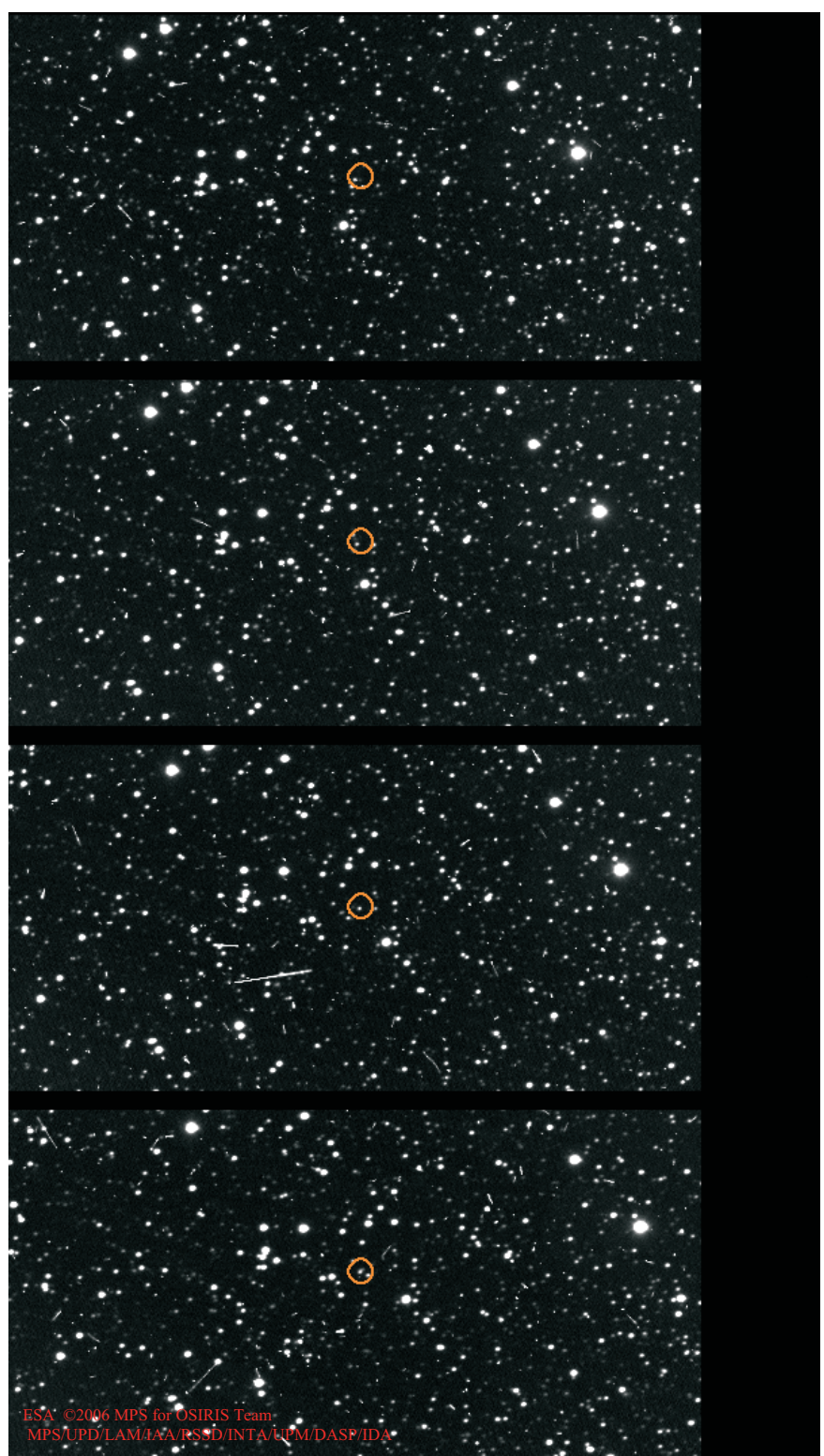

Fig. 2. Four images of asteroid (2867) Steins. The images in the second, third, and fourth panel, from bottom to top, were taken approximately 5, 10 , and $15 \mathrm{~h}$ after the first image, respectively. In all images Steins can be seen within the orange circle, demonstrating the excellent tracking of the spacecraft.

The average clear filter brightness of asteroid (2867) Steins was converted into absolute brightness. The image calibration provides the flux from Steins in $\mathrm{W} \mathrm{m}^{-2} \mathrm{~nm}^{-1}$ at a wavelength of $600 \mathrm{~nm}$, assuming a solar spectrum. First the flux was corrected for the $\approx 12.3 \%$ of the light that is outside the photometric aperture with two pixels radius due to the point spread function of the camera. Then the total flux was corrected for the spectrum of Steins being redder than that of the Sun using

$F_{\mathrm{c}}=F_{\mathrm{u}} \frac{\int_{\lambda} F_{\odot}(\lambda) T(\lambda) \mathrm{d} \lambda}{\int_{\lambda} F_{\text {steins }}(\lambda) T(\lambda) \mathrm{d} \lambda}$.

Here $F_{\mathrm{c}}$ and $F_{\mathrm{u}}$ are the corrected and uncorrected flux of asteroid Steins at $600 \mathrm{~nm}, T(\lambda)$ is the system throughput shown in Fig. 1, and $F_{\odot}(\lambda)$ and $F_{\text {steins }}(\lambda)$ are the spectra of the Sun and of Steins,

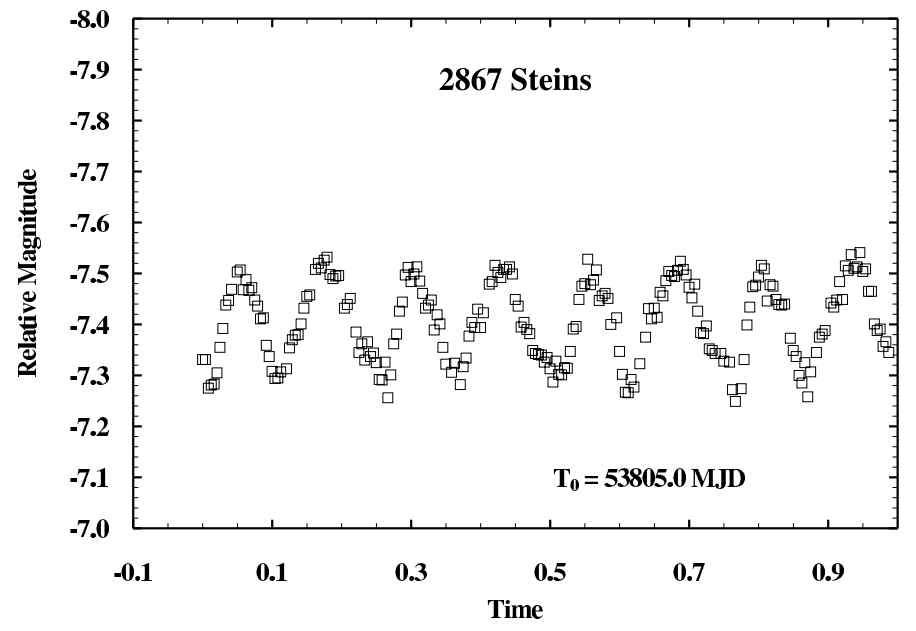

Fig. 3. All OSIRIS observations of asteroid Steins, covering four rotation periods.

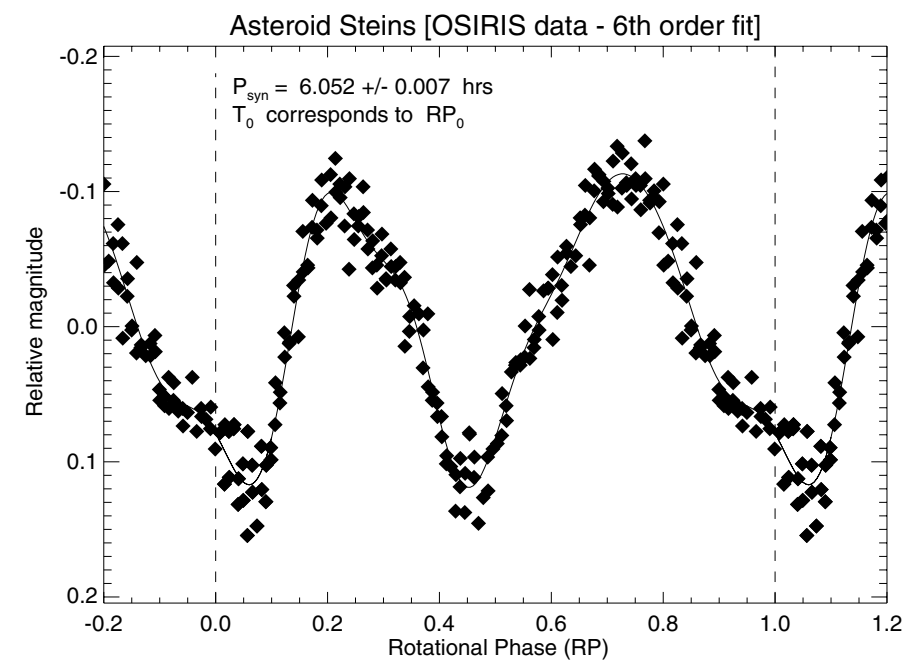

Fig. 4. The light curve of asteroid (2867) Steins from OSIRIS data. Magnitudes are given with arbitrary zero point. $T_{0}$ is 53804.99981 MJD, the data at phases less than zero and larger than one are repeated for clarity.

respectively, both normalized to unity at $600 \mathrm{~nm}$. The solar spectrum was taken from Burlov-Vasiljev et al. (1995) and BurlovVasiljev et al. (1998) in the visible range, and from Woods et al. (1996) in the ultraviolet range $(<350 \mathrm{~nm})$. The disk-center spectra from Burlov-Vasiljev et al. were corrected for limb darkening using the co-efficients of Neckel and Labs (1994). The spectrum of Steins was taken from Barucci et al. (2005). We assumed a constant albedo of Steins below $390 \mathrm{~nm}$ where, to our knowledge, no spectrum exists. Anyhow, the contribution of the ultraviolet spectral range to the flux measured through the clear filter is low.

$F_{\mathrm{c}}=(8.70 \pm 0.15) \times 10^{-18} \mathrm{~W} \mathrm{~m}^{-2} \mathrm{~nm}^{-1}$ is about $2.5 \%$ higher than $F_{\mathrm{u}}$. With $F_{\mathrm{c}}$ and the spectrum of Barucci et al. (2005) we know the absolute spectral flux distribution of Steins. Then the visual magnitude of Steins is given by:

$m_{V}=-2.5 \log \left(F_{\mathrm{c}} \int_{\lambda} F_{\text {steins }}(\lambda) T_{V}(\lambda) \mathrm{d} \lambda\right)+C$.

Here $T_{V}$ is the transmission of the standard $V$ filter taken from Bessel (1990). We determine the constant $C$ as the difference between the apparent magnitude of the Sun of -26.75 (Cox 2000) 
Table 1. Comparison of photometry of asteroid (2867) Steins. $H$ is the absolute magnitude with an assumed slope parameter of 0.15 .

\begin{tabular}{lccc}
\hline \hline & This work & ${\mathrm{H} 04^{a}}^{a}$ & ${\mathrm{~W} 05^{b}}^{b}$ \\
\hline Period [h] & $6.052 \pm 0.007$ & $6.06 \pm 0.05$ & $6.048 \pm 0.007$ \\
Amplitude [mag] & $0.23 \pm 0.03$ & 0.2 & $0.29 \pm 0.04^{c}$ \\
$H$ [mag] & $13.05 \pm 0.03$ & $13.18 \pm 0.04$ & $13.18 \pm 0.02$ \\
\hline
\end{tabular}

${ }^{a}$ Hicks et al. (2004); ${ }^{b}$ Weissman et al. (2005); ${ }^{c}$ in $R$ filter.

and the convolution of the solar spectrum with the $V$ filter as in Eq. (2). The apparent magnitude of asteroid (2867) Steins is $V(r, \Delta, \alpha)=16.61 \pm 0.03$. The corresponding magnitude at heliocentric and observer distance of $1 \mathrm{AU}$ would be $V(1,1$, $\alpha)=14.68$. The conversion to absolute magnitude depends on the phase function of the asteroid, parameterized with the slope parameter $G$ (Bowell et al. 1989). For the standard assumption of $G=0.15$, the absolute magnitude is $13.05 \pm 0.03$. With an albedo between 0.3 and 0.45 (Fornasier et al. 2006; Lamy et al. 2006), the absolute magnitude of 13.05 corresponds to an effective diameter between $4.8 \mathrm{~km}$ and $6.0 \mathrm{~km}$.

\section{Summary}

Table 1 summarizes the results of the present paper and compares them to the photometry of Hicks et al. (2004) and Weissman et al. (2005). The agreement on the rotation period is excellent.

The absolute brightness of (2867) Steins determined from the OSIRIS measurements is $\approx 13 \%$ higher than that derived from ground based measurements. The reason could be the photometric slope parameter $G$, which in E type asteroids is normally larger than the standard value of $0.15(\approx 0.5$, e.g. Harris et al. 1989b). Since the phase angle of (2867) Steins during the OSIRIS observations was larger than during the observations of Hicks et al. (2004) $\left(11.5^{\circ}\right)$ and Weissman et al. $(2005)\left(17^{\circ}\right)$, the data would be most consistent with $G \approx 0.3$ and an absolute brightness of $\approx 13.3$. Additionally, the absolute brightness of an asteroid with a light curve of moderate amplitude varies with aspect angle by a few tenth of a magnitude (Karttunen and Bowell 1989). Since the position of Steins on the sky differed by more than 90 degrees between the ground based observations from 2004 (Hicks et al. 2004; Weissman et al. 2005) and the OSIRIS observations in 2006, it is likely that the aspect angle was indeed different.
The nominal flyby with the asteroid Steins will take place on September 5th, 2008 at a velocity of $8.565 \mathrm{~km} \mathrm{~s}^{-1}$ and a close approach of $1745 \mathrm{~km}$. The obtained results from OSIRIS will be fundamental together with ground based observational campaigns to define the shape and pole orientation of the object. These properties will allow to optimize the spacecraft geometry during the Steins flyby to maximize the scientific return of the Rosetta mission.

Finally, these results attest to the excellent capabilites of the OSIRIS camera in terms both of scientific usefulness and technical performance.

Acknowledgements. The OSIRIS imaging system on board Rosetta is managed by the Max-Planck-Intitute for Solar System Research in Katlenburg-Lindau (Germany), thanks to an International collaboration between Germany, France, Italy, Spain, and Sweden. The support of the national funding agencies DLR, CNES, ASI, MEC, and SNSB is gratefully acknowledged. We acknowledge the work of the Rosetta Science Operations Centre at ESA/ESTEC and of the Rosetta Mission Operations Centre at ESA/ESOC who made these observations possible on short notation and operated the spacecraft. S.C.L. acknowledges support from the Leverhulme Trust. This research made use of JPL's online ephemeris generator (HORIZONS).

\section{References}

A'Hearn, M. A., Belton, M. J. S., Delamere, W. A., et al. 2005, Science, 310, 258

Barucci, M. A., Fulchignoni, M., Fornasier, S., et al. 2005, A\&A, 430, 313

Bessel, M. S. 1990, PASP, 102, 1181

Bowell, E., Hapke, B., Domingue, D., et al. 1989, Application of Photometric Models to Asteroids, in Asteroids II, ed. R. P. Binzel, T. Gehrels, \& M. D. Matthews (Tucson: Univ. of Arizona press), 524

Burlov-Vasiljev, K. A., Gurtovenko, E. A., \& Matvejev, Yu. B. 1995, Sol. Phys., 157, 51

Burlov-Vasiljev, K. A., Matvejev, Yu. B., \& Vasiljeva, I. E. 1998, Sol. Phys., 177, 25

Cox, A. N. 2000, Allen's astrophysical quantities (New York: AIP Press; Springer)

Fornasier, S., Belskaya, I., Fulchignoni, M., et al. 2006, A\&A, 449, L9

Harris, A. W., Young, J. W., Bowell, E., et al. 1989a, Icarus, 77, 171

Harris, A. W., Young, J. W., Contreiras, L., et al. 1989b, Icarus, 81, 365

Hicks, M. D., Bauer, J. M., \& Tokunaga, A. T. 2004, IAU Circ., 8315

Karttunen, H., \& Bowell, E. 1989, A\&A, 208, 320

Keller, H. U., Barbieri, C., Lamy. P., et al. 2006, Space Sci. Rev., in press

Lamy, P., Jorda, L., Fornasier, S., et al. 2006, BAAS, 38, 594

Neckel, H., \& Labs, D. 1994, Sol. Phys., 153, 91

Weissman, P. R., Lowry, S. C., \& Choi, Y. J. 2005, BAAS, 37, 644

Woods, T. N., Prinz, D. K., Rottman, G. J., et al. 1996, J. Geophys. Res., 101, 9541 
M. Küppers et al.: OSIRIS observations of Asteroid (2867) Steins, Online Material p 1

\section{Online Material}


M. Küppers et al.: OSIRIS observations of Asteroid (2867) Steins, Online Material p 2

Table 2. Flux from Asteroid Steins measured by OSIRIS. MJD is the modified Julian Date corrected for light-travel time, mag is the magnitude measured in the clear filter with arbitrary zero point. The letter $\mathrm{X}$ in the first column denotes measurements that are uncertain due to contamination by stars.

\begin{tabular}{|c|c|c|}
\hline & MJD & $\begin{array}{l}\text { Mag } \\
\end{array}$ \\
\hline & 53804.99981 & -7.331 \\
\hline & 53805.00395 & -7.331 \\
\hline & 53805.00812 & -7.275 \\
\hline & 53805.01228 & -7.281 \\
\hline & 53805.01645 & -7.283 \\
\hline & 53805.02062 & -7.305 \\
\hline & 53805.02478 & -7.355 \\
\hline & 53805.02895 & -7.392 \\
\hline & 53805.03312 & -7.438 \\
\hline & 53805.03728 & -7.447 \\
\hline & 53805.04145 & -7.469 \\
\hline \multirow[t]{17}{*}{$\mathrm{X}$} & 53805.04562 & -7.539 \\
\hline & 53805.04978 & -7.503 \\
\hline & 53805.05395 & -7.507 \\
\hline & 53805.05812 & -7.468 \\
\hline & 53805.06228 & -7.488 \\
\hline & 53805.06645 & -7.467 \\
\hline & 53805.07062 & -7.472 \\
\hline & 53805.07479 & -7.448 \\
\hline & 53805.07896 & -7.436 \\
\hline & 53805.08313 & -7.411 \\
\hline & 53805.08729 & -7.413 \\
\hline & 53805.09146 & -7.359 \\
\hline & 53805.09563 & -7.337 \\
\hline & 53805.09979 & -7.308 \\
\hline & 53805.10396 & -7.294 \\
\hline & 53805.10813 & -7.295 \\
\hline & 53805.11229 & -7.307 \\
\hline \multirow[t]{10}{*}{$X$} & 53805.11646 & -7.272 \\
\hline & 53805.12063 & -7.313 \\
\hline & 53805.12479 & -7.354 \\
\hline & 53805.12896 & -7.370 \\
\hline & 53805.13313 & -7.379 \\
\hline & 53805.13729 & -7.380 \\
\hline & 53805.14146 & -7.401 \\
\hline & 53805.14563 & -7.432 \\
\hline & 53805.14979 & -7.455 \\
\hline & 53805.15396 & -7.458 \\
\hline \multirow[t]{10}{*}{$X$} & 53805.15813 & -7.420 \\
\hline & 53805.16229 & -7.508 \\
\hline & 53805.16646 & -7.520 \\
\hline & 53805.17063 & -7.511 \\
\hline & 53805.17479 & -7.526 \\
\hline & 53805.17896 & -7.532 \\
\hline & 53805.18313 & -7.498 \\
\hline & 53805.18729 & -7.490 \\
\hline & 53805.19146 & -7.495 \\
\hline & 53805.19563 & -7.496 \\
\hline \multirow[t]{4}{*}{$\mathrm{X}$} & 53805.19979 & -7.567 \\
\hline & 53805.20396 & -7.432 \\
\hline & 53805.20813 & -7.439 \\
\hline & 53805.21229 & -7.451 \\
\hline \multirow[t]{9}{*}{$X$} & 53805.21646 & -7.328 \\
\hline & 53805.22064 & -7.385 \\
\hline & 53805.22479 & -7.345 \\
\hline & 53805.22896 & -7.362 \\
\hline & 53805.23313 & -7.330 \\
\hline & 53805.23729 & -7.366 \\
\hline & 53805.24146 & -7.337 \\
\hline & 53805.24563 & -7.344 \\
\hline & 53805.24979 & -7.325 \\
\hline
\end{tabular}

Table 2. Continued.

\begin{tabular}{|c|c|c|}
\hline & MJD & Mag \\
\hline & 53805.25396 & -7.292 \\
\hline & 53805.25813 & -7.291 \\
\hline & 53805.26229 & -7.326 \\
\hline & 53805.26646 & -7.256 \\
\hline & 53805.27063 & -7.301 \\
\hline & 53805.27479 & -7.362 \\
\hline & 53805.27896 & -7.381 \\
\hline & 53805.28313 & -7.426 \\
\hline & 53805.28729 & -7.444 \\
\hline & 53805.29146 & -7.497 \\
\hline & 53805.29563 & -7.512 \\
\hline & 53805.29979 & -7.484 \\
\hline & 53805.30396 & -7.499 \\
\hline & 53805.30813 & -7.513 \\
\hline & 53805.31229 & -7.485 \\
\hline & 53805.31646 & -7.461 \\
\hline & 53805.32063 & -7.432 \\
\hline & 53805.32479 & -7.438 \\
\hline & 53805.32896 & -7.448 \\
\hline & 53805.33313 & -7.389 \\
\hline & 53805.33729 & -7.419 \\
\hline & 53805.34146 & -7.401 \\
\hline & 53805.34563 & -7.355 \\
\hline & 53805.34979 & -7.322 \\
\hline$X$ & 53805.35396 & -7.501 \\
\hline & 53805.35813 & -7.306 \\
\hline & 53805.36229 & -7.324 \\
\hline$X$ & 53805.36646 & -7.688 \\
\hline & 53805.37063 & -7.282 \\
\hline & 53805.37479 & -7.317 \\
\hline & 53805.37896 & -7.334 \\
\hline & 53805.38313 & -7.377 \\
\hline & 53805.38731 & -7.404 \\
\hline & 53805.39146 & -7.394 \\
\hline & 53805.39563 & -7.430 \\
\hline & 53805.39979 & -7.394 \\
\hline & 53805.40396 & -7.423 \\
\hline$X$ & 53805.40813 & -7.549 \\
\hline & 53805.41229 & -7.479 \\
\hline & 53805.41646 & -7.484 \\
\hline & 53805.42063 & -7.516 \\
\hline & 53805.42479 & -7.502 \\
\hline & 53805.42896 & -7.492 \\
\hline & 53805.43313 & -7.508 \\
\hline & 53805.43729 & -7.508 \\
\hline & 53805.44146 & -7.513 \\
\hline & 53805.44563 & -7.499 \\
\hline & 53805.44979 & -7.449 \\
\hline & 53805.45396 & -7.436 \\
\hline & 53805.45813 & -7.395 \\
\hline & 53805.46229 & -7.404 \\
\hline & 53805.46646 & -7.390 \\
\hline & 53805.47063 & -7.382 \\
\hline & 53805.47479 & -7.349 \\
\hline & 53805.47896 & -7.343 \\
\hline & 53805.48313 & -7.341 \\
\hline & 53805.48729 & -7.340 \\
\hline & 53805.49146 & -7.326 \\
\hline & 53805.49563 & -7.335 \\
\hline & 53805.49979 & -7.313 \\
\hline & 53805.50396 & -7.287 \\
\hline & 53805.50813 & -7.328 \\
\hline & 53805.51229 & -7.302 \\
\hline & 53805.51646 & -7.301 \\
\hline & 53805.52063 & -7.315 \\
\hline
\end{tabular}


M. Küppers et al.: OSIRIS observations of Asteroid (2867) Steins, Online Material p 3

Table 2. Continued.

\begin{tabular}{|c|c|c|}
\hline & MJD & Mag \\
\hline & 53805.52479 & -7.314 \\
\hline & 53805.52896 & -7.347 \\
\hline & 53805.53313 & -7.391 \\
\hline & 53805.53729 & -7.396 \\
\hline & 53805.54146 & -7.449 \\
\hline & 53805.54563 & -7.475 \\
\hline & 53805.54979 & -7.480 \\
\hline & 53805.55396 & -7.528 \\
\hline & 53805.55813 & -7.478 \\
\hline & 53805.56229 & -7.487 \\
\hline & 53805.56646 & -7.507 \\
\hline & 53805.57063 & -7.447 \\
\hline & 53805.57479 & -7.456 \\
\hline & 53805.57896 & -7.461 \\
\hline & 53805.58313 & -7.451 \\
\hline & 53805.58729 & -7.400 \\
\hline \multirow[t]{8}{*}{$\mathrm{X}$} & 53805.59146 & -7.512 \\
\hline & 53805.59563 & -7.413 \\
\hline & 53805.59979 & -7.347 \\
\hline & 53805.60396 & -7.302 \\
\hline & 53805.60813 & -7.267 \\
\hline & 53805.61229 & -7.266 \\
\hline & 53805.61646 & -7.292 \\
\hline & 53805.62063 & -7.277 \\
\hline \multirow[t]{2}{*}{$\mathrm{X}$} & 53805.62479 & -7.504 \\
\hline & 53805.62896 & -7.323 \\
\hline \multirow[t]{26}{*}{$X$} & 53805.63313 & -7.563 \\
\hline & 53805.63729 & -7.375 \\
\hline & 53805.64146 & -7.431 \\
\hline & 53805.64563 & -7.411 \\
\hline & 53805.64979 & -7.432 \\
\hline & 53805.65396 & -7.414 \\
\hline & 53805.65813 & -7.463 \\
\hline & 53805.66229 & -7.456 \\
\hline & 53805.66646 & -7.486 \\
\hline & 53805.67063 & -7.504 \\
\hline & 53805.67479 & -7.496 \\
\hline & 53805.67896 & -7.494 \\
\hline & 53805.68313 & -7.506 \\
\hline & 53805.68729 & -7.524 \\
\hline & 53805.69146 & -7.508 \\
\hline & 53805.69563 & -7.497 \\
\hline & 53805.69979 & -7.473 \\
\hline & 53805.70396 & -7.452 \\
\hline & 53805.70813 & -7.479 \\
\hline & 53805.71229 & -7.426 \\
\hline & 53805.71647 & -7.384 \\
\hline & 53805.72064 & -7.382 \\
\hline & 53805.72479 & -7.397 \\
\hline & 53805.72896 & -7.352 \\
\hline & 53805.73313 & -7.349 \\
\hline & 53805.73729 & -7.343 \\
\hline \multirow[t]{3}{*}{$X$} & 53805.74146 & -7.487 \\
\hline & 53805.74563 & -7.343 \\
\hline & 53805.74979 & -7.328 \\
\hline$X$ & 53805.75396 & -7.550 \\
\hline
\end{tabular}

Table 2. Continued.

\begin{tabular}{|c|c|c|}
\hline & MJD & Mag \\
\hline & 53805.75813 & -7.326 \\
\hline & 53805.76229 & -7.272 \\
\hline & 53805.76646 & -7.249 \\
\hline \multirow[t]{17}{*}{$\mathrm{X}$} & 53805.77063 & -7.521 \\
\hline & 53805.77479 & -7.274 \\
\hline & 53805.77896 & -7.331 \\
\hline & 53805.78313 & -7.399 \\
\hline & 53805.78729 & -7.434 \\
\hline & 53805.79146 & -7.474 \\
\hline & 53805.79563 & -7.477 \\
\hline & 53805.79979 & -7.493 \\
\hline & 53805.80396 & -7.516 \\
\hline & 53805.80813 & -7.509 \\
\hline & 53805.81229 & -7.446 \\
\hline & 53805.81646 & -7.478 \\
\hline & 53805.82063 & -7.475 \\
\hline & 53805.82479 & -7.449 \\
\hline & 53805.82896 & -7.439 \\
\hline & 53805.83313 & -7.438 \\
\hline & 53805.83729 & -7.440 \\
\hline \multirow[t]{9}{*}{$\mathrm{X}$} & 53805.84146 & -7.549 \\
\hline & 53805.84563 & -7.373 \\
\hline & 53805.84979 & -7.349 \\
\hline & 53805.85396 & -7.337 \\
\hline & 53805.85813 & -7.300 \\
\hline & 53805.86229 & -7.285 \\
\hline & 53805.86646 & -7.325 \\
\hline & 53805.87063 & -7.258 \\
\hline & 53805.87479 & -7.307 \\
\hline \multirow[t]{26}{*}{$\mathrm{X}$} & 53805.87896 & -7.603 \\
\hline & 53805.88313 & -7.345 \\
\hline & 53805.88729 & -7.375 \\
\hline & 53805.89146 & -7.381 \\
\hline & 53805.89564 & -7.388 \\
\hline & 53805.90396 & -7.442 \\
\hline & 53805.90813 & -7.434 \\
\hline & 53805.91229 & -7.448 \\
\hline & 53805.91646 & -7.484 \\
\hline & 53805.92063 & -7.449 \\
\hline & 53805.92479 & -7.515 \\
\hline & 53805.92896 & -7.506 \\
\hline & 53805.93313 & -7.537 \\
\hline & 53805.93729 & -7.510 \\
\hline & 53805.94146 & -7.513 \\
\hline & 53805.94563 & -7.541 \\
\hline & 53805.94979 & -7.504 \\
\hline & 53805.95396 & -7.509 \\
\hline & 53805.95813 & -7.465 \\
\hline & 53805.96229 & -7.465 \\
\hline & 53805.96646 & -7.401 \\
\hline & 53805.97063 & -7.388 \\
\hline & 53805.97479 & -7.391 \\
\hline & 53805.97896 & -7.357 \\
\hline & 53805.98313 & -7.366 \\
\hline & 53805.98729 & -7.345 \\
\hline
\end{tabular}

